\title{
COSTEO BASADO EN ACTIVIDADES -ABC: REVISIÓN DE LITERATURA
}

\author{
Activity-based costing $-A B C$ : literature review
}

\author{
Alina Marcela Bustamante Salazar \\ M.Sc. en Administración. Instituto Tecnológico Metropolitano. Medellín, Colombia. \\ alinabustamante@itm.edu.co
}

\section{Cómo citar / How to cite}

Bustamante, A. (2015). Costeo basado en actividades. Revisión de la literatura. Revista CEA, 1(1), 109-119.

Recibido: 24 de junio de 2014

Aceptado: 16 de septiembre de 2014

\section{Resumen}

En la década de 1980 la contabilidad de gestión experimenta el surgimiento de un nuevo sistema: costeo basado en actividades, lo que implica un cambio de énfasis del registro de información para la valuación de los inventarios y la elaboración de informes financieros, al control de los recursos, las actividades y la generación de información para la toma de decisiones. Este artículo presenta una síntesis de los antecedentes, los fundamentos, las potencialidades, las limitaciones y las aplicaciones en sectores específicos del costeo basado en actividades, a partir de la revisión de literatura de artículos publicados en revistas académicas especializadas durante el periodo 2007-2014.

La revisión muestra que a pesar de las potencialidades de los sistemas $A B C$ para la asignación más precisa de los costos, el control de los recursos y la gestión de la capacidad disponible, la complejidad y el alto costo que implica su implementación se constituyen en limitaciones. Así mismo, se evidencia una mayor aplicación en organizaciones privadas del sector salud y manufactura, encontrándose menos evidencias respecto a su aplicación en organizaciones públicas.

Palabras clave: costeo basado en actividades, sistemas de costos, ABC.

\begin{abstract}
In the 1980, management accounting experience the emergence of a new system: activity-based costing and with it a change of emphasis, the record of information for the valuation of inventories and reporting financial; control of resources, activities and generating information for decision-making. This article presents an overview of the background, rationale, potential, limitations and applications in specific sectors of activity-based costing, based on the literature review of articles published in academic journals during the 2007-2014 period.
\end{abstract}

The review shows that despite the potential of $A B C$ systems for more accurate cost allocation, resource control and management of the available capacity; the complexity and high cost of its implementation constitute limitations. Likewise, a wide application in private health organizations and manufacturing sector is evident while it is less obvious regarding its application in public organizations.

Keywords: activity-based costing, cost systems, ABC. 


\section{INTRODUCCIÓN}

De modo general, la contabilidad puede ser comprendida como un sistema de información que mide y comunica hechos económicos y sociales de una organización, permitiendo a los usuarios tomar decisiones informadas acerca del uso de los recursos y el cumplimiento de los objetivos de la organización (Aguiar et al., 1998). Tal como lo señalan Ripoll Feliu y Urquidi (2010, p. 3), esta se constituye en el sistema de información más importante de la empresa, que opera en los ámbitos interno y externo, para satisfacer las necesidades de diferentes usuarios. Las empresas modelan, desarrollan y utilizan diferentes sistemas de información contables (Gómez, 2007), uno de ellos es el de la contabilidad de gestión.

Según Johnson y Kaplan (1988) la contabilidad de gestión apareció por primera vez en el siglo XIX en los Estados Unidos, con el propósito de determinar con precisión el costo de los productos y contribuir con información para la fijación de los precios de venta, la valuación de los inventarios y apoyar la toma de decisiones. Sin embargo, al priorizar la aplicación de normas financieras y fiscales para cumplir requerimientos legales, de usuarios externos, se obnubilaron las potencialidades de esta para medir la eficiencia, la rentabilidad y apoyar la implementación de la estrategia de la empresa.

Durante casi todo el siglo XX la contabilidad de gestión atraviesa un proceso de estancamiento (Johnson \& Kaplan, 1988), (Torrecilla, Fernández, \& Gutiérrez, 1993) hasta llegar a la década de 1980, con la aparición del sistema de costos basado en las actividades $-\mathrm{ABC}-$, y con él un cambio de énfasis, del registro de información para la valuación de los inventarios y la elaboración de informes financieros; al control de los recursos, las actividades y la generación de información para la toma de decisiones.
Así como lo señala Mallo (2009, p. 21), el surgimiento de los costos $A B C$ representa para la contabilidad de gestión un cambio de paradigma; varios autores coinciden en considerar que estos se constituyen en una de las áreas de investigación y de aplicación más importante en la contabilidad de gestión (Rodríguez-Maeso, 2010), (Duque Roldán \& Osorio Agudelo, 2013), (Harris \& Durden, 2012), (Fei \& Isa, 2010), (Osorio Agudelo \& Duque Roldán, 2002).

Este artículo se propone explicar los antecedentes, la fundamentación, las potencialidades, las limitaciones y los factores que inciden en la implementación del costeo $A B C$, así como la aplicación en sectores específicos, a partir de la revisión de literatura de artículos publicados en revistas académicas durante el periodo 2007-2014.

\section{METODOLOGÍA}

La revisión de la literatura que se presenta en este artículo fue efectuada en la base académica Ebsco, utilizando como criterio de búsqueda los conceptos activity-based costing y $A B C$, en los títulos y en las palabras clave de los artículos publicados en el periodo 2007 hasta mayo de 2014. También se incluyeron algunos libros y las memorias de congresos de contabilidad y gestión que se encuentran en google académico.

En total se revisaron 51 referencias, estas fueron clasificadas en tres categorías: aquellas que estudian los antecedentes y fundamentación del costeo $A B C$ (dentro de esta categoría se incluyeron algunos artículos de revisión de literatura sobre contabilidad de gestión; los costos $A B C$ son una de las temáticas de mayor relevancia de este campo académico) que corresponde al $43 \%$ de las referencias revisadas; el $37 \%$ se refiere a las potencialidades, limitaciones y factores que inciden en la implementación de este sistema de costos; y un $20 \%$ corresponde a la 
aplicación del costeo $A B C$ en sectores específicos.

Aunque existen algunos estudios de revisión sobre contabilidad de gestión (João Lunkes, Ripoll Feliu, \& Silva da Rosa, 2013), (Duque Roldán \& Osorio Agudelo, 2013), (Harris \& Durden, 2012); (Ripoll Feliu \& Urquidi, 2010) y de costos ABC (Moisello, 2012), (Fei \& Isa, 2010), (Rodríguez-Maeso, 2010), (Caldera, Baujín, Ripoll Feliu, \& Vega, 2007), el alcance y los periodos de revisión son diferentes a los que se presentan en este artículo.

La investigación de João Lunkes et al. (2013) analiza los artículos sobre contabilidad de gestión publicados durante el periodo 20002010 en revistas académicas de España y Brasil indexadas en ISI y SCOPUS. Los autores encuentran que los costos son una de las líneas de investigación más importantes en contabilidad de gestión, sin embargo su estudio es general y no hace énfasis en los costos $A B C$.

Una situación similar se presenta en el estudio de Ripoll Feliu y Urquidi (2010) los autores investigan las herramientas de contabilidad de gestión más utilizadas en la práctica empresarial, los hallazgos indican que los sistemas de costos $A B C$ son una nueva técnica de gestión que se utiliza en algunas empresas. Duque Roldán y Osorio Agudelo (2013) analizan el estado de la investigación en contabilidad de gestión y costos en Colombia y concluyen que este es incipiente; las revistas colombianas especializadas en contabilidad y administración presentan una baja indexación, aunque reconocen que los sistemas de costos $A B C$ son uno de los temas que más interesa a los investigadores.

Desde el punto de vista de los costos $A B C$, Rodríguez-Maeso (2009) presenta una revisión de literatura de artículos publicados durante el periodo 2003-2009 en revistas académicas de alto impacto, incluidas en los índices Journal Citation Report-JCR y Scimago Journal Rank-SJR. El autor indaga sobe las áreas de investigación, los fundamentos teóricos y los métodos de investigación aplicados y concluye que los factores contextuales y políticos inciden en la implementación del $A B C$, los errores en las estimaciones de tiempo y la información para la toma de decisiones son los temas más investigados.

Las revisiones anteriores sobre contabilidad de gestión y los sistemas de costos $A B C$ presentan limitaciones en cuanto a los temas incluidos, el periodo de tiempo revisado y los criterios de selección de artículos. Los artículos que revisan la evolución de la contabilidad de gestión sirven de contexto general para conocer las principales líneas de investigación que interesan a los académicos de esta área; una de ellas se refiere, precisamente, a los sistemas de costos $A B C$, sin embargo, los autores no estudian en profundidad los desarrollos de estos sistemas.

Aunque algunos autores han estudiado la evolución (Caldera et al., 2007) y los factores de éxito en la implementación de los sistemas ABC (Moisello, 2012), (Fei \& Isa, 2010), el alcance de su revisión es limitada en el tiempo, solo incluyen la bibliografía publicada hasta 2009 y en otros casos (Rodríguez-Maeso, 2010) solo tienen en cuenta los artículos publicados en revistas académicas de alto impacto, incluidas en ISI y SCOPUS, lo cual excluye un gran número de revistas académicas y profesionales del área contable y administrativa que se encuentran indexadas en otras bases de datos. Las consideraciones anteriores se constituyen en argumentos que justifican la realización de una nueva revisión.

\section{RESULTADOS Y DISCUSIÓN}

Los artículos y textos revisados fueron organizados en tres temáticas: aquellos que estudian los antecedentes y la 
fundamentación de los sistemas de costos $A B C$; las potencialidades, las limitaciones y los factores que inciden en la implementación de estos sistemas $y$, finalmente, aquellos que documentan su aplicación en sectores específicos. A continuación se explican cada uno.

\section{Antecedentes y fundamentación de los sistemas de costos $A B C$}

Los antecedentes del costeo $A B C$ se pueden encontrar en la obra de Porter (1987) acerca de la cadena de valor, y en el artículo de Miller y Vollmann (1985) sobre el costeo de transacciones. La cadena de valor se concibe como el conjunto interrelacionado de actividades creadoras de valor que se desarrollan internamente en la empresa, para entregar un producto o servicio al cliente - perspectiva micro-, así como en los eslabones de la cadena a la cual pertenece la empresa - perspectiva macro- Se requiere, entonces, estructurar apropiadamente los procesos de la empresa, identificando las actividades que originan los costos, por medio de relaciones causa efecto asociadas en la cadena de valor (Kapić, 2014), (Caldera et al., 2007, p. 16), (Rodríguez, 2001, p. 17).

De otro lado, Miller y Vollmann (1985) consideran que un adecuado control de los costos indirectos parte del estudio y el control de las transacciones que los originan. De modo que se requiere, inicialmente, conocer los factores que originan los costos en cada departamento, es decir, las transacciones que consumen recursos, para posteriormente asignarlas a los productos o servicios (Johnson \& Kaplan, 1988, pp. 228229).

Los sistemas de costos $A B C$ se definen como un sistema de gestión empresarial que permite a las organizaciones calcular el costo de las actividades y los procesos con base en los recursos que estas utilizan y luego los asigna a los productos, los servicios y demás objetos de costos, según los requerimientos de la gerencia, suministrando información relevante para la toma de decisiones en cuanto a la medición del desempeño, el control de los procesos, el presupuesto de los recursos y el análisis de la rentabilidad de estos, los servicios, los clientes y los proveedores (Cooper \& Kaplan, 1992, 1998).

El marco de acción del enfoque basado en las actividades se extiende, más allá del cálculo de costos, a otros procesos de las organizaciones, surgiendo así la gestión basada en actividades - activity-based management $-A B M-$ (Kaplan \& Cooper, 1999) , la contabilidad por actividades activity accounting $-A A-(B r i m s o n, 1997)$ y el presupuesto basado en actividades activity-based budgeting - ABB- (Cataldo Pizarro, 1997). Cuervo Tafur y Osorio Agudelo (2007) definen la gestión basada en actividades como una metodología administrativa para la planeación y la gestión, que facilita la evaluación del desempeño de las actividades que integran la operación del negocio y los recursos que estas emplean. Por su parte, Brimson (1997) afirma que la contabilidad por actividades identifica y analiza las actividades que se ejecutan en una organización, determina su costo y su rendimiento. Este análisis permite a los gerentes comprender detalladamente qué hace la empresa y cómo lo hace, y ayuda a identificar aquellas actividades que no agregan valor y que pueden eliminarse para optimizar el uso de los recursos. Por último, el presupuesto basado en actividades permite determinar los recursos que requieren las unidades operativas y los centros de responsabilidad, basándose en las demandas de actividades que se espera que se realicen (Kaplan \& Cooper, 1999, p. 347) (Kaplan \& Anderson, 2007, p. 21).

Los sistemas de costos $A B C$ parten de una visión sistémica de la organización, esta se entiende como un conjunto de procesos y 
actividades interrelacionadas que se llevan a cabo para cumplir el objeto social. Las actividades consumen recursos y son necesarias para fabricar los productos 0 prestar los servicios que oferta la organización, de modo que los recursos se asignan a las actividades, y el costo de las actividades se asigna a los objetos de costos a través de bases de asignación, factores que causan el costo denominados inductores o cost drivers. Así entonces, los recursos, las actividades y los inductores de costos son los conceptos básicos que fundamentan los sistemas de costos $A B C$.

Inicialmente se desarrolló el sistema de costeo basado en las actividades -ABC. Thomas Johnson, Robert Kaplan y Robin Cooper fueron los autores pioneros que construyeron las bases conceptuales de este sistema. Tal como lo señala Mallo (2009), el boom publicitario ayudó a su rápida difusión en los contextos gerenciales y contables de muchos países. Sin embargo, con el tiempo se experimentó una disminución y una vuelta atrás en las empresas que habían iniciado su implementación. El alto costo, la rigidez dificultades para actualizar el sistema- y la complejidad en su implementación y sostenimiento se constituyeron en razones para su decadencia.

Posteriormente, Robert Kaplan y Steven Anderson proponen una nueva versión denominada costeo basado en el tiempo invertido por actividad o TDABC, por sus siglas en inglés. Según lo señala Kaplan (2014, p.78) la innovación de esta nueva versión es redescubrir la ecuación básica en el cálculo de los costos, según la cual, el costo de un recurso es igual a la cantidad de unidades de recursos utilizados por el precio de cada unidad de recurso. Este sistema permite asignar los recursos directamente a los objetos de costos, a través de un coeficiente de capacidad que se calcula dividiendo el costo de los recursos sobre su capacidad práctica, es decir, el tiempo que estos están disponibles en la organización, para su posterior asignación a los objetos de costo (Gómez \& Quintero, 2012).

El costeo basado en el tiempo invertido por actividad, utiliza el tiempo como inductor para la asignación de los costos, y permite actualizar fácilmente el cálculo de los costos, cada vez que se realicen cambios en los métodos o procedimientos de trabajo.

\section{Potencialidades, limitaciones y factores que inciden en la implementación de los sistemas de costos $A B C$}

Los sistemas de costos $A B C$ surgen en la década de 1980 como una alternativa para hacer frente a las limitaciones de los sistemas tradicionales de costos para la asignación precisa y confiable de los costos indirectos. Los sistemas tradicionales se habían enfocado principalmente en la valoración de los inventarios y la determinación de los costos de venta para la elaboración de informes financieros, en algunas ocasiones se utilizaban criterios arbitrarios para la asignación de los costos a cada línea de producto, esto dificultaba el análisis de la rentabilidad de los productos y poco contribuía en la toma de decisiones.

Así entonces, las principales potencialidades de los sistemas de costos $A B C$ están relacionadas con la asignación precisa de los costos, la utilización de múltiples bases de asignación, inductores o cost drivers para la asignación de estos. Por otra parte, el análisis de las actividades realizadas en la empresa ayuda a comprender el funcionamiento de la misma y a detectar las posibilidades de mejoramiento, eliminando las actividades que no agregan valor. De este modo, estos sistemas se constituyen en una herramienta gerencial para evaluar el desempeño de la empresa y mejorar su posición competitiva. Algunos autores, como Maiga y Jacobs (2008), han encontrado que el uso extensivo de los sistemas de costos 
$A B C$ están asociados positivamente con el mejoramiento de la calidad, el costo, los ciclos de tiempo y la rentabilidad de las empresas.

En relación con las limitaciones de los sistemas de costos $A B C$, algunos autores consideran que el diseño y la implementación de estos sistemas suelen ser complejas y costosas, dado que se requiere recolectar información detallada acerca de las actividades que realizan los empleados, los tiempos y los recursos que se utilizan en estas (Cuervo Tafur \& Osorio Agudelo, 2007), (Hedhili, 2013). Para superar esta debilidad, Kaplan y Anderson (2007) proponen los sistema de costos basados en el tiempo invertido por actividad o TDABC, según los autores «la innovación del modelo de TDABC es que utiliza el tiempo para asignar costos directamente de los recursos a los objetos de coste, eliminando por completo la tediosa fase de asignación de costos de los recursos a las actividades» (Kaplan \& Anderson, 2007, p.28).

Así mismo, en algunas empresas resulta problemático y costoso el sostenimiento y continuidad del sistema de costos $A B C$, por la cantidad de datos que requiere procesar y analizar, unido a la falta de conexión con otros sistemas de información de la empresa (Gómez, Duque Roldán, \& Cuervo Tafur, 2005), (Kaplan \& Anderson, 2007), (Cokins \& Căpuşneanu, 2011), (López-Mejía, GómezMartínez, \& Marín-Hernández, 2011).

Del mismo modo, la selección y construcción de los direccionadores o cost drivers es un proceso complejo que requiere un amplio conocimiento de la metodología y de la organización, así como sistemas de información que faciliten la recolección y análisis de los datos (Duque Roldán, Cuervo Tafur, \& Gómez, 2005), (Răvaş \& Monea, 2009).

En empresas multiproceso y multiproducto se hace necesario subdividir las actividades para reflejar las diferencias en el consumo de recursos y asignar correctamente los costos a los productos, lo cual implica aumentar la complejidad en la estructura -mayor número de actividades - y el cálculo de costos (Patxi Ruiz De Arbulo-López, FortunySantos, Vintró-Sánchez, \& Basañez-Llantada, 2013, p. 235), (Kaplan \& Anderson, 2004).

Por otra parte, es difícil de adaptar el sistema a los cambios en los métodos y procedimientos de trabajo, se presentan condiciones de incertidumbre que puede hacer variar las condiciones sobre las cuales se calculó el costo de las actividades; para incorporar estos cambios se requiere modificar la estructura de costos y esto demanda tiempo y recursos (Hedhili, 2013, p. 172), (Raeesi \& Amini, 2013, p. 43).

Con el propósito de perfeccionar y lograr mejores resultados en la implementación de los sistemas $A B C$, autores como Hossein Mortaji, Bagherpour y Mazdeh (2013), Karakas, Koyuncu, Erol y Kokangul (2010) han propuesto la utilización de la lógica difusa para la estimación de la información requerida en estos sistemas, como los tiempos de las actividades y la capacidad práctica.

Algunos autores se han interesado en estudiar los factores que determinan el éxito - fracaso de la implementación de los sistemas de costos $A B C$. Al respecto, Fei e Isa (2010) construyen una estructura de factores comportamentales-relacionados con la disponibilidad de recursos y la incidencia en la evaluación de desempeño-; organizacionales - principalmente relativos al apoyo de la gerencia y la capacitación-; técnicos -identificación de actividades, selección de inductores-; y otros relacionados con la cultura y la estructura organizacional -orientación a resultados, trabajo en equipo, centralización- que determinan el éxito de la implementación de los sistemas $A B C$.

Por otra parte, Moisello (2012) estudia los factores de proceso y de contexto que 
inciden en las implementaciones exitosas. En relación con los primeros, la autora encuentra que el apoyo de la gerencia, la disponibilidad de recursos y la vinculación de los sistemas $A B C$ como componente de la evaluación de desempeño inciden de forma positiva en la implementación. Así mismo, la incidencia del sistema $A B C$ en la determinación de los salarios y la calidad del sistema de información son factores contextuales que favorecen o disminuyen la probabilidad de éxito.

Alcouffe, Berland y Levant (2008) consideran los sistemas de costos $A B C$ como una innovación de la contabilidad de gestión y utilizan la teoría del Actor-Red para explicar el éxito en la difusión de esta innovación. Los canales de comunicación y difusión de los sistemas de costos $A B C$ inciden positivamente en la decisión de implementación (Alcouffe \& Guedri, 2008).

Desde una perspectiva política, Englund y Gerdin (2008) concluyen que los procesos de implementación de los sistemas $A B C$ no son neutrales, en las organizaciones existen relaciones de poder e intereses divergentes que inciden en el éxito o el fracaso de la implementación. Por otra parte, Arena y Solle (2008) consideran los sistemas ABC y $A B M$ como sistemas de control de gestión, que involucran aspectos cognitivos aprendizaje- y sociales -interacción entre diferentes miembros de la organizaciónque inciden en los procesos de implementación.

Otras condiciones relacionadas con el desconocimiento del sistema de costos $A B C$, el temor a que se aumente el trabajo o se descubran las ineficiencias, pueden conducir al fracaso de la implementación (Moisello, 2012).

En síntesis, son múltiples los factores que inciden en el éxito o fracaso en la implementación de los sistemas de costos $A B C$, la planificación, la capacitación del personal que participa en el proceso y el liderazgo de las personas que dirigen la implementación, pueden contribuir a vencer la resistencia al cambio y promover la participación y compromiso de los actores de la organización, para obtener resultados positivos.

\section{Aplicación de los sistemas de costos $A B C$ en sectores específicos}

En la literatura revisada se puede identificar algunos artículos que documentan la aplicación de los sistemas de costos $A B C$ en sectores específicos, especialmente en organizaciones de ofrecen servicios de salud, energía y, empresas manufactureras del sector de componentes para automóviles y metalmecánicas.

En relación con los sistemas de costos en el sector de la salud, varios autores (Gómez, Duque-Roldan \& Carmona, 2008) y (Mendoza, 2013) coinciden en considerar los costos $A B C$ y los sistemas de costos basados en el tiempo invertido por actividad Kaplan (2014), Kaplan y Porter (2011) como sistemas de costeo pertinentes para estas organizaciones. Estos ayudan a identificar aspectos a mejorar relacionados con el uso eficiente de los recursos, tiempos de atención al paciente, sustitución de recursos, costo de la capacidad no utilizada, entre otros. De modo que, el reto en las organizaciones del sector salud consiste en medir con exactitud los costos y lograr reducirlos, sin sacrificar el resultado en los tratamientos médicos de los pacientes.

La investigación de Miranda, De Carvalho, Martins, y De Faria acerca de la utilización de los sistemas de costos $A B C$ en hospitales universitarios de Brasil, concluye que, a pesar de las potencialidades de este sistema para las organizaciones del sector salud, su utilización es limitada por la complejidad del mismo y porque los sistemas de información 
utilizados suplen las necesidades de la organización.

Maria and Nicoleta (2011) estudian la aplicabilidad del sistema de costos $A B C$ en el sector de energía, y establecen las condiciones que deben tenerse en cuenta para su implementación, las autoras consideran que este sistema permiten una clasificación más amplia de los costos, no solo desde su naturaleza económica sino, además, en términos de su destinación, en cuanto a la producción, el suministro y el transporte.

En relación con los sistemas de costos en el sector de energía es importante mencionar que en Colombia es obligatorio el uso de este sistema de costeo para las empresas de servicios públicos domiciliarios, que ofrecen servicios de acueducto, alcantarillado, aseo, energía y gas combustible, con fundamento en la Resolución 1417 de abril de 1997 de la Superintendencia de Servicios Públicos Domiciliarios, y para las empresas de telecomunicaciones según la Resolución 2863 de octubre de 1996 (Osorio Agudelo \& Duque Roldán, 2002).

La industria de componentes para automóviles se caracteriza por ser multiproceso, por la variedad de productos que fabrica y por los estrechos márgenes de rentabilidad, lo cual implica una gestión de costos más rigurosa. Algunos autores (Mariana, 2013), (Patxi Ruiz De Arbulo-López et al., 2013), (Patxi Ruiz De Arbulo-López \& Fortuny-Santos, 2011) han estudiado la aplicación de los sistemas de costos $A B C$ en este industria y concluyen que estos sistemas son pertinentes para el cálculo preciso de los costos, además la aplicación del costeo basado en el tiempo invertido por actividad permite reflejar la heterogeneidad de los procesos y productos que se fabrican y suministran información acerca de la capacidad no utilizada.

Los estudios de López-Mejía et al. (2011), Stout y Propri (2011), Öker y Adigüzel (2010), documentan la aplicación de los sistemas de costos $A B C$ en medianas empresas de industrias manufactureras de diversos componentes metalmecánicos. López-Mejía et al. (2011) presenta un estudio de caso de una empresa mexicana, los autores encuentran que la necesidad de disponer de una información de costos más precisa y confiable y mejorar la competitividad de la empresa son las razones que motivaron la implementación del sistema. Así mismo, Ríos, Muñoz y Rodríguez (2014) estudian la implementación de los sistemas de costos $A B C$ en las pymes mexicanas; las evidencias señalan que aunque los empresarios reconocen el potencial de la metodología para ayudar a la toma de decisiones, identificar y suprimir actividades que no agregan valor, su tasa de aplicación es muy baja, en opinión de las autoras, es necesario una mayor divulgación de la metodología de costeo $A B C$.

\section{CONCLUSIONES}

Los sistemas de costos $A B C$ siguen siendo una de las líneas de investigación más importantes de la contabilidad de gestión. La literatura sobre el tema es abundante, especialmente aquella que estudia los antecedentes y la fundamentación, las potencialidades, las limitaciones y los factores que inciden en la implementación de estos sistemas. Se encuentra alguna literatura que documenta la aplicación en sectores específicos, especialmente en organizaciones de servicios-salud, energía - y en empresas manufactureras del sector privado de países desarrollados.

Tal como lo señala Fei e Isa (2010) se han realizado pocos trabajos empíricos sobre la implementación de los sistemas de costos $A B C$ en empresas de países en desarrollo. Así mismo, falta estudiar la aplicabilidad de estos sistemas en organizaciones públicas, pues aunque en algunos países como 
Colombia existen leyes que obligan la adopción de estos sistemas por parte de las entidades públicas, no se encontraron evidencias suficientes que documenten las experiencias relativas con su implementación. Futuras investigaciones podrían abordar estos asuntos.

\section{REFERENCIAS}

Aguiar, H., Cadavid, L. A., Cardona, J., Carvalho, J., Jiménez, J., \& Upegui, M. E. (1998). Diccionario de términos contables para Colombia. Medellín: Editorial Universidad de Antioquia.

Alcouffe, S., Berland, N., \& Levant, Y. (2008). Actor-networks and the diffusion of management accounting innovations: A comparative study. Management Accounting Research, 19(1), 1-17. doi: http://dx.doi.org/10.1016/j.mar.2007.04.0 01

Alcouffe, S., \& Guedri, Z. (2008). Le rôle des canaux de communication et des caractéristiques perçues de l'innovation dans le processus d'adoption de la comptabilité par activités (méthode $A B C$ ). Comptabilité - Contrôle - Audit, 14, 39-66.

Arena, L., \& Solle, G. (2008). Apprentissage Organisationnel et Contrôle de Gestion: Une lecture possible de I'ABC/ABM? Comptabilité - Contrôle - Audit, 14, 67-85.

Brimson, J. A. (1997). Contabilidad por actividades. Un enfoque de costes basado en las actividades. México: Alfaomega Marcombo.

Caldera, J., Baujín, P., Ripoll Feliu, V. M., \& Vega, V. (2007). Evolución en la configuración de los sistemas de costeo basado en las actividades. Actualidad Contable FACES, 10(14), 13-28.

Cataldo Pizarro, J. (1997). Gestión del presupuesto $A B C$. Su integración con la calidad y las normas ISO 9000. México: AlfaomegaMarcombo.
Cokins, G., \& Căpuşneanu, S. (2011). Sustaining an Effective ABC/ABM. System. Theoretical \& Applied Economics, 18(2), 47-58.

Cooper, R., \& Kaplan, R. S. (1992). Activity-Based Systems: Measuring the Costs of Resource Usage. Accounting Horizons, 6(3), 1-13.

Cooper, R., \& Kaplan, R. S. (1998). The Promise-And Peril--Of Integrated Cost Systems. Harvard Business Review, 76(4), 109-119.

Cuervo Tafur, J., \& Osorio Agudelo, J. A. (2007). Costeo basado en actividades -ABC-. Gestión basada en actividades -ABM-. Bogotá: Ecoe Ediciones.

De Arbulo-López, P. R., \& Fortuny-Santos, J. (2011). Innovación en gestión de costes: del ABC al TDABC. Dirección $y$ Organización, 43, 16-26.

De Arbulo-López, P. R., Fortuny-Santos, J., VintróSánchez, C., \& Basañez-Llantada, A. (2013). Aplicación de time-driyen activity-based costing en la producción de componentes de automóvil. Dyna, 88(2), 234-240. doi: $10.6036 / 5105$

Duque Roldán, M. I., Cuervo Tafur, J., \& Gómez, L. F. (2005). Los direccionadores: ¿'solución o problema del ABC? Contaduría Universidad de Antioquia, 47, 39-58.

Duque Roldán, M. I., \& Osorio Agudelo, J. A. (2013). Estado Actual de la investigación en Costos y Contabilidad de Gestión en Colombia.

Englund, H., \& Gerdin, J. (2008). Transferring knowledge across sub-genres of the $A B C$ implementation literature. Management Accounting Research, 19(2), 149-162. doi: http://dx.doi.org/10.1016/j.mar.2008.01.0 02

Fei, Z. Y., \& Isa, C. R. (2010). Factors Influencing Activity-Based Costing Success: A Research Framework. International Journal of Trade, Economics and Finance, 1(2), 144-150.

Gómez, L., Duque-Roldán, M. y Carmona, J. (2008). La información contable y de costos en las entidades de salud: una herramienta para su transformación. Contaduría Universidad de Antioquia, 53, 145-172. 
Gómez, L. F., Duque Roldán, M. I., \& Cuervo Tafur, J. (2005). Gestión de riesgos en el costeo basado en actividades: una alternativa para su implantación exitosa. Contaduría - Universidad de Antioquia, 47, 61-85.

Gómez, L. F., \& Quintero, J. B. (2012). Conveniencia de la utilización del time driven activity bases costing TDABC. Paper presentado al XVII Congreso Internacional de Contaduría, Administración e Informática, México.

Gómez, M. (2007). Comprendiendo las relaciones entre los sistemas contables, los modelos contables y los sistemas de información contables empresariales. Revista Internacional Legis de Contabilidad \& Auditoría, 32, 235-253.

Harris, J., \& Durden, C. (2012). Management Accounting Research: An Analysis of Recent Themes and Directions for the Future. Journal of Applied Management Accounting Research, 10(2), 21-41.

Hedhili, N. (2013). Le positionnement de la méthode «du temps requis pour exécuter les opérations» ou "time driven activity based costing» (TD $A B C$ ) par rapport à la méthode $A B C$ (comptabilité à base d'activité). Revue des Sciences de Gestion, 48(263/264), 171-177.

Hossein Mortaji, S. T., Bagherpour, M., \& Mazdeh, M. M. (2013). Fuzzy Time-Driven Activity-Based Costing. Engineering Management Journal, 25(3), 63-73.

João Lunkes, R., Ripoll Feliu, V. M., \& Silva da Rosa, F. (2013). Study of Published Articles on Management Accounting in Brazil and Spain. Revista Contabilidade \& Finanças USP, 24(61), 11-26.

Johnson, T., \& Kaplan, R. S. (1988). La Contabilidad de costes. Auge y caída de la contabilidad de gestión. Barcelona: Plaza \& Janes.

Kapić, J. (2014). Activity Based Costing - ABC. Business Consultant / Poslovni Konsultant, 6(32), 9-16.
Kaplan, R. S. (2014). Improving value with TDABC. HFM -Healthcare Financial Management, 68(6), 76.

Kaplan, R. S., \& Anderson, S. (2007). What-If Analysis and Activity-Based Budgeting. Forecasting Resource Demands. TimeDriven Activity-Based Costing: A simpler and More Powerful Path to Higher Profits. Boston: Harvard Business Press.

Kaplan, R. S., \& Anderson, S. R. (2004). TimeDriven Activity-Based Costing. Harvard Business Review, 82(11), 131-138.

Kaplan, R. S., \& Anderson, S. R. (2007). The Speed-Reading Organization. Business Finance, 13(6), 39-42.

Kaplan, R. S., \& Cooper, R. (1999). Coste y Efecto. Cómo usar el $A B C$, el $A B M$ y el $A B B$ para mejorar la gestión, los procesos y la rentabilidad (Segunda ed.). Barcelona: Gestión 2000.

Kaplan, R. S., \& Porter, M. E. (2011). How to Solve The Cost Crisis In Health Care. Harvard Business Review, 89(9), 46-64.

Karakas, E., Koyuncu, M., Erol, R., \& Kokangul, A. (2010). Fuzzy programming for optimal product mix decisions based on expanded ABC approach. International Journal of Production Research, 48(3), 729-744. doi: 10.1080/00207540802471249

López-Mejía, M. R., Gómez-Martínez, A., \& MarínHernández, S. (2011). Sistema de costos $A B C$ en la mediana empresa industrial mexicana. Cuadernos de Contabilidad, 12(30), 23-43.

Maiga, A. S., \& Jacobs, F. A. (2008). Extent of ABC Use and Its Consequence. Contemporary Accounting Research, 25(2), 533-566.

Mallo, C. (2009). El nuevo/viejo paradigma: Costes basados en el tiempo invertido por Actividad. Partida Doble, 19(211), 20-35.

Rof, L. M., \& Farcane, N. (2011). Current State and Evolution Perspectives for Management Accounting In The Energy Sector By Implementing The ABC Method. Annals of the University of Oradea, Economic Science Series, 20(1), 653-660. 
Radu, M. (2013). Modernizing Management Accounting By The ABC Method. Internal Auditing \& Risk Management, 8(4), 1-11.

Mendoza, R. (2013). Administración de los costos en instituciones de la salud. Administration costs in health institutions. Incae Business Review, 2(7), 2-6.

Miller, J. G., \& Vollmann, T. E. (1985). The hidden factory. Harvard Business Review, 63(5), 142-150.

Moisello, A. M. (2012). ABC: Evolution, Problems of Implementation and Organizational Variables. American Journal of Industrial and Business Management, 2, 55-63.

Öker, F., \& Adigüzel, H. (2010). Time-driven activity-based costing: An implementation in a manufacturing company. Journal of Corporate Accounting \& Finance (Wiley), 22(1), 75-92. doi: 10.1002/jcaf.20646

Osorio Agudelo, J. A., \& Duque Roldán, M. I. (2002). Modelos de medición y desempeño interrelacionados en la Contabilidad de Gestión. Contaduría Universidad de Antioquia, 41, 113-143.

Porter, M. E. (1987). Ventaja competitiva: creación y sostenimiento de un desempeño superior. México: Compañía Editorial Continental.

Raeesi, R., \& Amini, A. (2013). Developing A Grey Activity Based Costing (G-ABC) Method To Capture The Inherent Uncertainty In Identifying Cost Drivers' Consumption Rates. Academy of Accounting \& Financial Studies Journal, 17(2), 41-56.

Răvaş, B., \& Monea, A. (2009). The definiton and determination of the cost drivers, basis elements in the abc system implemented in a tourism unit. Lucrari Stiintifice, 11(4), 1-6.
Ríos, M., Muñoz, C. \& Rodríguez, M. (2014). Is the activity based costing system a viable instrument for small and medium enterprises? The case of Mexico. Estudios Gerenciales, 30, 220-232.

Ripoll Feliu, V. M., \& Urquidi, A. (2010). Herramientas de contabilidad de gestión utilizadas en la práctica empresarial: una revisión crítica de los trabajos de investigación. Academia, Revista Latinoamericana de Administración, 44, 120.

Rodríguez-Maeso, J. E. (2010). El actual estado del arte en los sistemas $\mathrm{ABC} / \mathrm{M}$ : un análisis e interpretación de la literatura internacional. En línea: http://www.observatorioiberoamericano.org/

Rodríguez, G. (2001). Diferentes métodos de costeo frente a la Throughput Accounting (TA) de la Teoría de las Restricciones (TOC). Cuadernos de Contabilidad, 12, 1433.

Stout, D. E., \& Propri, J. M. (2011). Implementing Time-Driven Activity-Based Costing at a Medium-Sized Electronics Company. Management Accounting Quarterly, 12(3), 1-11.

Torrecilla, A., Fernández, A., \& Gutierrez, G. (1993). Contabilidad de costes y contabilidad de gestión. Volumen 1. Madrid: McGraw-Hill. 